\title{
Comprehensive 3-D Imaging of Near Field Magnetic Coil Patterns Using Multiple 2-D Planar Slices
}

\author{
Aparicio Carranza ${ }^{1}$, Reena Dahle, PhD $^{2}$, Kevin Hart ${ }^{2}$ and Sean Hart ${ }^{2}$ \\ ${ }^{1}$ New York City College of Technology of The City University of New York (CUNY) \\ Brooklyn, New York, USA \\ acarranza@citytech.cuny.edu \\ ${ }^{2}$ State University of New York (SUNY) at New Paltz \\ New Paltz, New York, USA \\ dahler@engr.newpaltz.edu; hartk1@hawkmail.newpaltz.edu; harts3@hawkmail.newpaltz.edu
}

\begin{abstract}
Wireless power transfer through near field magnetic coupling is becoming increasingly popular in modern electronics. Consequently, coil geometry design is being examined in order to optimize wireless power transfer efficiency and coil-to-coil alignment sensitivity. This paper presents a Python program that was written to generate a full 3-dimensional (3-D) near field radiation pattern from a planar array of magnetic field probes. Measurements were taken with the Coil Under Test (CUT) at different heights and then interpolated by the software to generate the 3-D image. The EMScan system used to measure the magnetic fields had a limited resolution making it difficult for the user to clearly analyzer coil behavior. To improve the voxel resolution, dynamic 3-D convolutional image interpolation was performed. A Graphical User Interface is developed to allow the user to view 2-dimensional (2-D) slices of the image along three orthogonal planes, in addition to a full 3-D pattern. The software also allows a user to upload a photograph of the coil that was measured, which is segmented to extract the region of the image that contains the conductor. The resulting outline can be overlaid with the radiation patterns so that the relationship between the coil geometry and the radiation pattern can be analyzed in detail.
\end{abstract}

Keywords: Image Processing, Image Interpolation, 3-D Imaging, Image Segmentation, Wireless Power Transfer, Near-Field Radiation Patterns.

\section{Introduction}

An abundance of research is being conducted on wireless power transfer due to its usability for many novel applications. These include wireless charging of consumer electronics such as cell phones and laptops, as well as wireless charging of parked cars to reduce fossil fuel emissions [1]. Due to the inherent inefficiency and skew sensitivity present in wireless charging, additional innovation is needed to optimize coil designs in terms of their geometry. Near field patterns embed a large amount of complexity, and as a result sophisticated display is necessary to compare the fields rendered from different coil geometries including those explored in [2] and [3]. The system described in this paper uses image processing to provide an interactive and comprehensive display of near field radiation patterns. There are many applications in which obtaining accurate near-field radiation pattern measurements is a critical step. The authors in [4] perform near field radiation pattern measurements on commercially-used antennas to help specify the exclusion zones in which humans risk unsafe levels of exposure to electromagnetic radiation. Papers [5]-[7] describe the measurement of the near field region along an arbitrary closed surface so that far field radiation patterns can be predicted. This method is preferable for antennas at lower frequency, like the parabolic reflector that was measured in [5], because directly obtaining far field radiation patterns necessitates outdoor measurements, which imposes undesirable factors such as multipath distortion and dynamic weather. Additionally, the authors in [8] created a high resolution near-field scanning system that is tasked with measuring the magnetic-near-field noise inherent to RFIC chips. All of the aforementioned literature involves the use of a single magnetic field probe that can move with three degrees of freedom to obtain measurements. A single probe minimizes probe interference but introduces mechanical complexity because of the necessary actuation and vibrational stabilization. The EMScan measurement system discussed in this paper uses a 2-D magnetic field probe array to provide an efficient and cost-effective design feedback on coils intended for wireless power transfer [9]. 
This project performs 3-D image interpolation where the third dimension is spatial variation of the field with distance from the plane of the Coil Under Test (CUT). This variation is important because the functional range in many of these applications is critical, and it is the immediate direction of magnetic field (H-Field) propagation.

Due to the fact that a significant amount of experimentation is being conducted to optimize near field patterns with respect to coil geometries, it was important to allow the user to overlay an image of the CUT with the patterns. Viewing this overlay aids in the development of an intuition on the dependence of near field patterns on coil geometry. To implement this, a significant amount of image segmentation and scaling was performed on a photograph of the CUT.

This paper provides an overview of the image processing carried out, as well as the functionality of the software created. Generated radiation patterns overlaid with the outline of the CUT are shown to display the utility of the software. Python was employed to develop all of the programming presented including use of its tkinter library to render a Graphical User Interface (GUI), Numeric Python for mathematical functions, and Matplotlib for image display. A MDL technologies EMSCAN EHX+ near field scanner was used to obtain near field measurements.

\section{Obtaining the Measurements}

The EMSCAN EHX+ measurement system embeds an array of magnetic field (H-field) probes and is typically used to measure the magnetic fields induced from a printed circuit board (PCB) thereby detecting the possibility of ElectroMagnetic Interference (EMI) [9]. For this purpose, measurement along a single plane close to the PCB is sufficient. To extend the measurement capabilities to a third dimension, multiple stands were manufactured using 3-D printing, so that the radiation patterns could be measured along parallel planes at different heights. These stands were manufactured at heights of 0.508 $\mathrm{mm}, 1.27 \mathrm{~mm}, 2.54 \mathrm{~mm}, 3.81 \mathrm{~mm}, 5.08 \mathrm{~mm}$ and $12.7 \mathrm{~mm}$. The height distribution included more resolution at smaller heights because near-field patterns are more dynamic near the plane of the coil, rendering interpolation to be more viable the further the measurement plane is from the CUT. A measurement taken with the $12.7 \mathrm{~mm}$ stand is shown in Fig. 1.

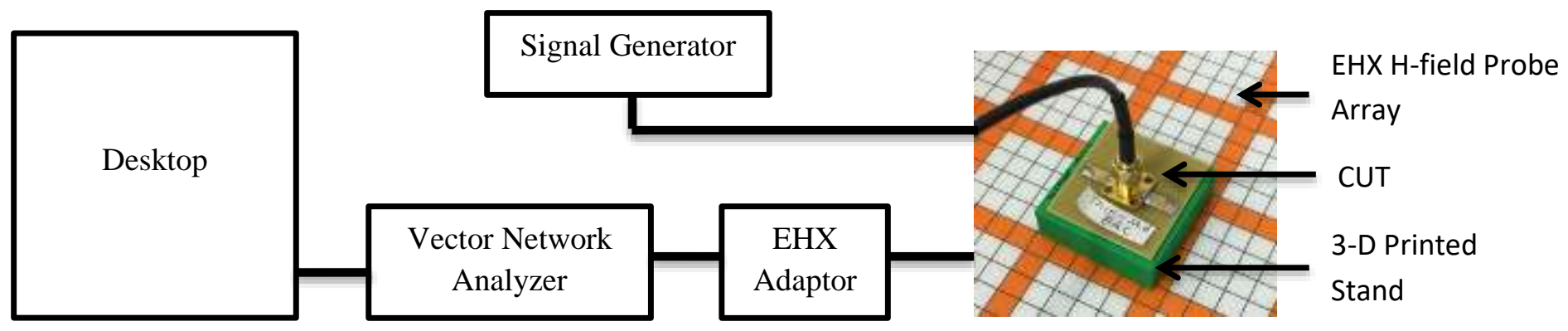

Fig. 1: Near-Field radiation measurement setup at $12.7 \mathrm{~mm}$.

The software that was developed extracts the raw data from the measurement along each plane while associating the corresponding height via user input. This data is formatted in a text file, which the software iterates through until it finds which magnetic field probes were enabled. It creates a 2-D matrix with the same size as the enabled probe array in which the value sensed by each probe is stored. This matrix represents the raw planer image, and these were then stacked along the third dimension in order of ascending heights to represent the raw 3-D image, which was ready for interpolation.

\section{Radiation Pattern Interpolation}

The original readings formed a 28 by 28-pixel array, resulting in only a total of 784 pixels in the raw image. Additionally, measurements were only obtained at 6 different heights. As a result, it was difficult to discern the radiation pattern of each CUT from its corresponding image. The goal was to resize the image and interpolate in between pixels in order to obtain a smoother and more coherent image using the theory presented in [10]. Interpolation was also carried out in between the measured plains so that spatial characteristics of the near field pattern could be analyzed as a function of distance from the CUT. It should be noted that this application is intended for use in computers with moderate processing power in a lab or classroom environment, and consequently care was taken in order to ensure that the code was not too computationally rigorous. The resolution of the interpolation can be increased to fulfill more powerful processing. 
Convolutional interpolation was selected due to its simplicity and relatively fast processing time. Bi-Linear, Bi-cubic, and Sinc kernels were tested within the project in terms of accuracy and processing time [11]. It was found that the significantly longer processing time present when using Bi-cubic and Sinc kernels did not justify their use, due to the fact that little difference was observed between their resulting image, and the image obtained under linear interpolation. Generation of the three-dimensional linear interpolation kernel was implemented using the code:

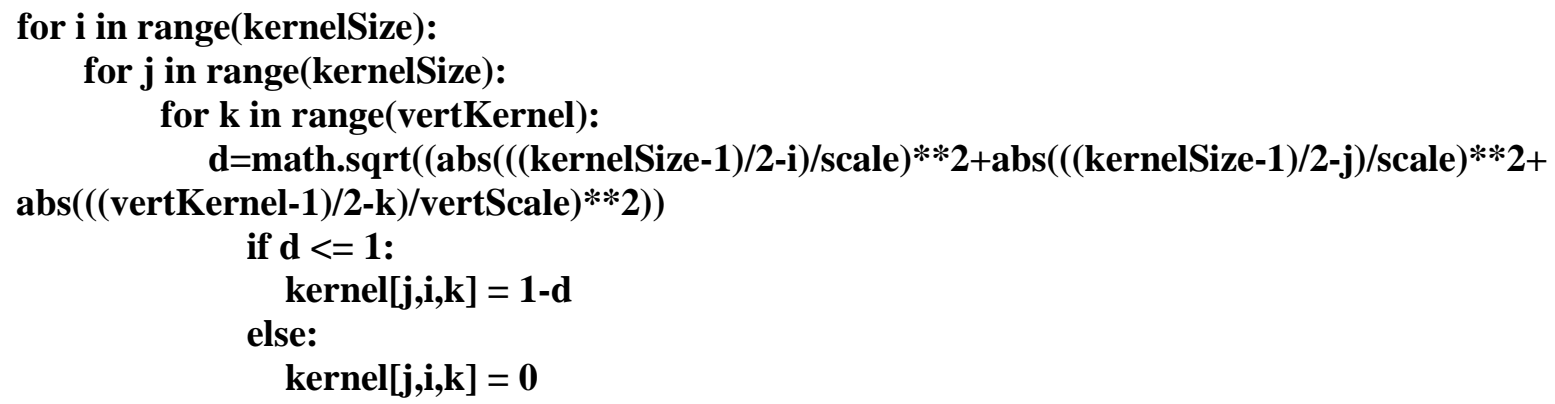

With [12] as a reference, NumPy, SciPy, and Matplotlib objects were used to program this project. In the above code, variable $d$ is defined as a pixel's distance from the center of the kernel, relative to the unscaled image. Additionally, zeroes were injected systematically between each of the unscaled 2-D slices by a scaling factor in order to increase the array's size, and to prepare the array for convolution. Following this, zero arrays were also padded in equal increments between the 2-D slices from the $1.27 \mathrm{~mm}$ pattern to the $5.08 \mathrm{~mm}$ pattern. Because of uneven spacing, the injection between the $0.508 \mathrm{~mm}$ slice and the $1.27 \mathrm{~mm}$ slice; as well as between the $5.08 \mathrm{~mm}$ and $12.7 \mathrm{~mm}$ slice were scaled separately and also interpolated individually to uphold even increments between the planes. Following convolution, aliasing was present in the form of patterns observed in the image due to unequal weighting between pixels during the operation. To atone for this, an array of ones was generated to be the same size as the unscaled image to act as normalizing factors for the result. All operations described above were repeated for the image of ones. When the interpolated image was divided by the normalizing array the aliasing was erased due to the fact that non-unity values in the normalized array corresponded to unequal weighting after convolving with the kernel. Finally, it is important to observe that all of the image processing algorithms implemented were designed to be scalable such that the user of the system can specify the amount of scaling desired in each dimension in order to fit analysis and system constraints.

\section{Coil Detection}

In order to allow the user to easily overlay an outline of the CUT with its radiation pattern and observe the relationship between the two, the software allows the user to upload a photograph of the coil. The code to create this was developed with the help of [12] and [13]. The picture of the coil is converted to a binary image that is true where a copper trace is detected, and false where the substrate or the background is detected. The first step of the segmentation process employed by the software is to use the canny edge detection algorithm on a version of the image that is smoothed by a Gaussian kernel with a large standard deviation, so that the rectangular outline of the substrate is detected, while the edges of coil are not because they were faded out. Next, a loop detection algorithm that was developed is used to obtain the indices of the edges that make up the largest closed loop in the image, which represents the outline of the substrate. Because the size of the substrate is known by the user, the vertices of these indices were able to be used to ensure proper scaling of the image.

Once the outline was detected, the image could be cropped so that it only included the substrate and the copper trace. Observation of the spiral coils in Fig. 1 shows that any regard for proximity in the segmentation would be obsolete because of the complexity of the contours. Therefore, color-based thresholding was used. This method imposed a challenge because the color of the copper traces was very close to the color of the substrate, so no color coordinate system conveyed a significant variation along a single dimension. This meant that spatial lighting variations in the picture would disrupt the thresholding if it was performed across the entire image. To account for this, the image was partitioned into multiple rectangular regions that each shared an edge with the substrate outline. In each region, the color coordinates of pixels near the edge of the substrate outline were averaged together to represent a known color for the substrate. The remaining pixels in each region were thresholded based on the distances in three-dimensional color coordinates between each pixel and the substrate value, 
and between each pixel and a lighter shade of the substrate value that was determined experimentally. Once this was done, binary dilation followed by binary erosion with the same structure was used to engulf the impurities in the region such as soldering errors. Some of the resulting binary images are shown in Fig. 2.
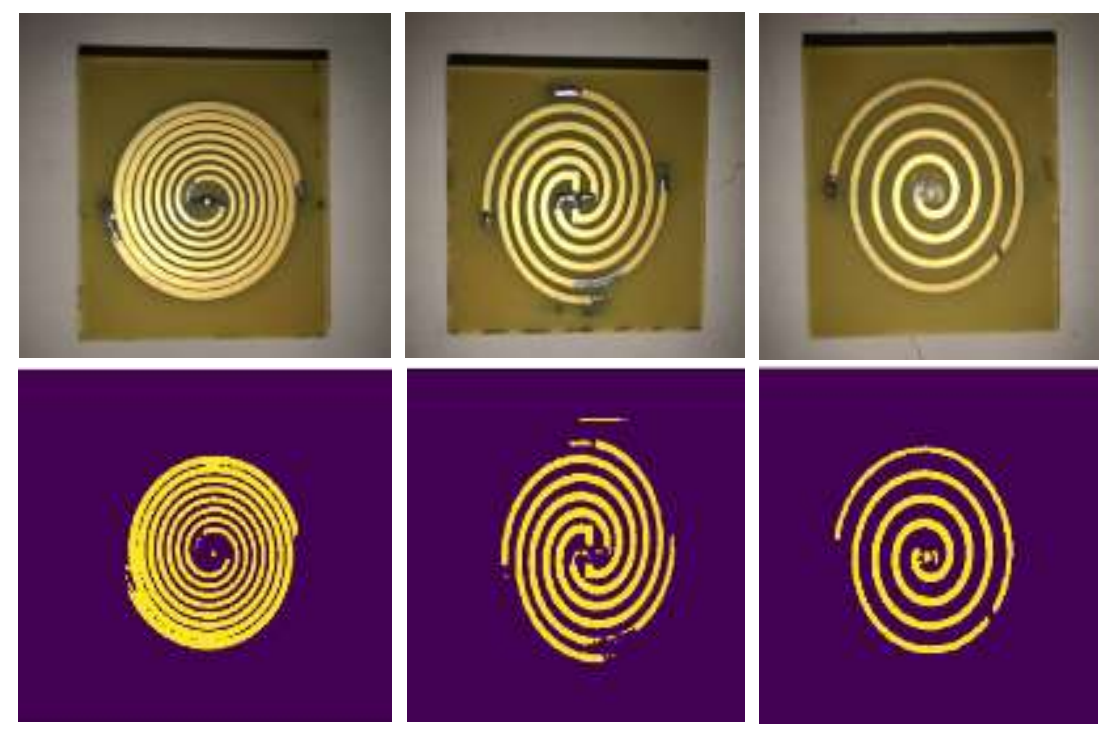

Fig. 2: Results of image segmentation for different spiral coils.

\section{System Interface and Results}

The raw image obtained from the EMScan machine is compared with the resulting interpolated image in Fig. 3 . All images generated are colormapped using Python's 'jet', which scales gradually between red, yellow, and blue where dark red represents a one and dark blue represents a zero. Comparison of the two images presented in Fig. 3 shows the enhanced readability that the interpolation provides. In addition, the interpolation can be scaled to raw images of greater resolution to facilitate a more accurate result.
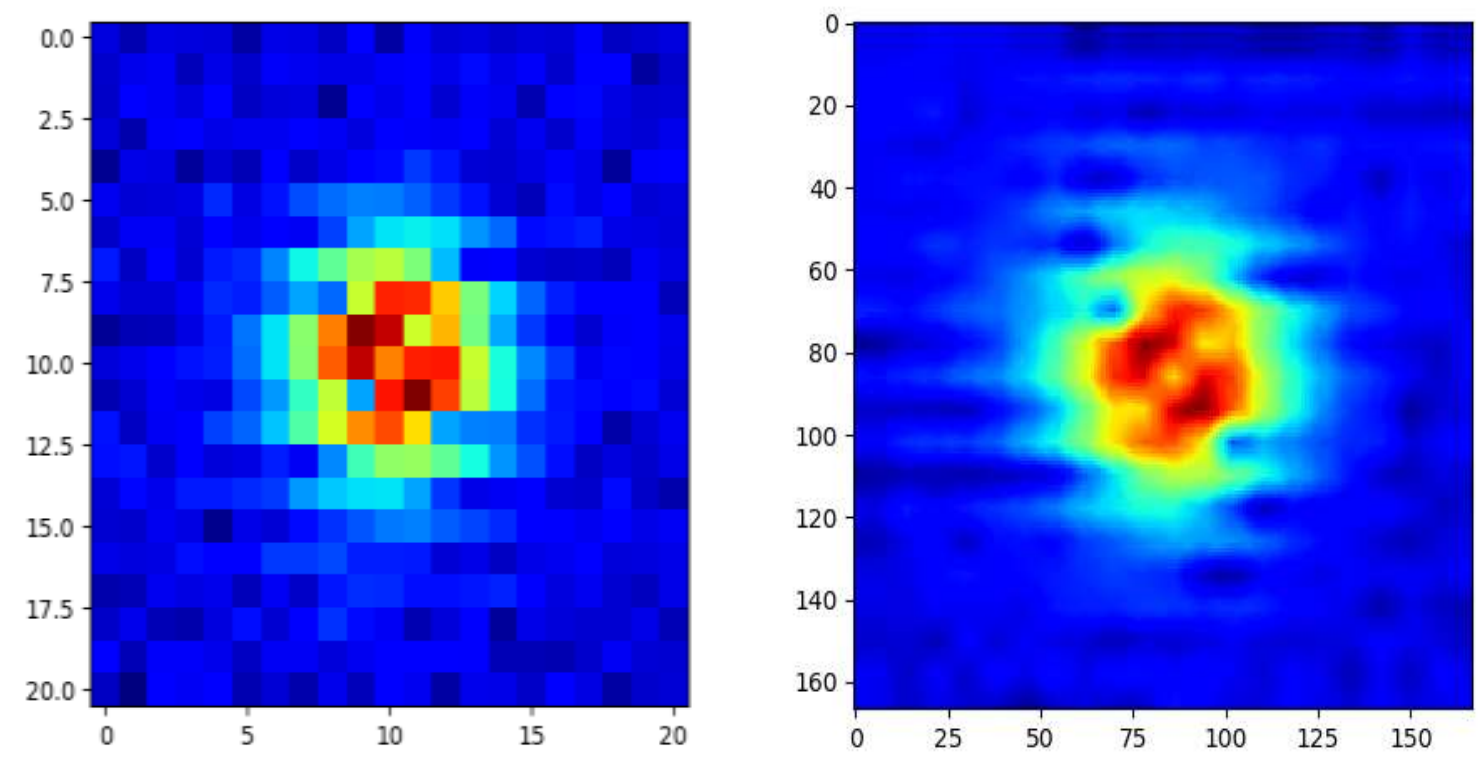

Fig. 3: 2-D interpolation of near field pattern. 
Specifically, measuring a larger spiral will allow the pattern to be represented by more H-field probes, which will result in a more accurate representation of the near field rendered by the spiral. The software also includes a scroll bar, which allows a user to navigate through different slices of the pattern corresponding to different vertical distances away from the CUT, which is similar to image display techniques employed in medical image viewing. This was implemented by indexing the third dimension of the 3-D matrix with the value returned by the scrollbar.

Moreover, the interface created allows users to scroll through 2-D slices of each of the dimensions within the plane of the CUT (along the $\mathrm{x}$ - and y-plane). This allows a user to view cross sections of the beam formed by the spiral, as shown in Fig. 4.
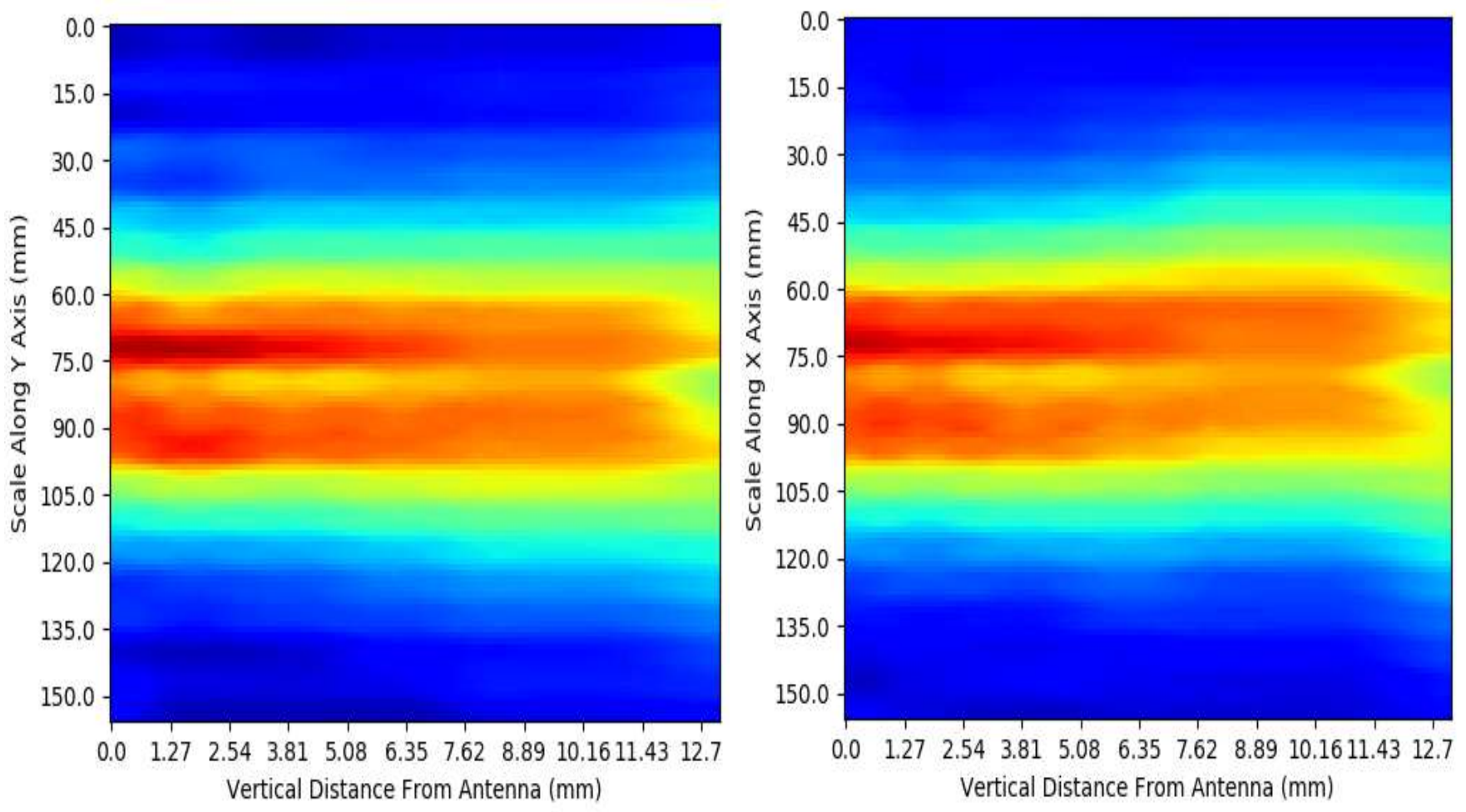

Fig. 4: Vertical slices of near field pattern along both dimensions of coils plane ( $\mathrm{x}$ and $\mathrm{y}$-plane).

The interface for the graphing in Fig. 4 also includes a scrollbar which allows a user to navigate through different planes of the critical dimension. It is important to note that the scaling along the horizontal axis is enlarged to highlight pattern variation that is observed when traveling away from the coil.

If the user uploads an image of the CUT, the software has the capability to overlay a properly scaled version of the coil segment's skeleton with the radiation pattern measurement taken along a parallel plane to the coil. This scaling was done by resizing the image by a factor represented with the ratio of the physical length of each pixel in the photograph to the length of each pixel in the interpolated radiation pattern along both dimensions. Use of these factors was made possible with knowledge of the dimensions of the cutout of the substrate and the size of the magnetic field probes on the measurement system. Following the resizing, this scaled image was padded with zeros for proper centering and overlaid with the radiation pattern measurement. The result of this feature is shown in Fig. 5. 


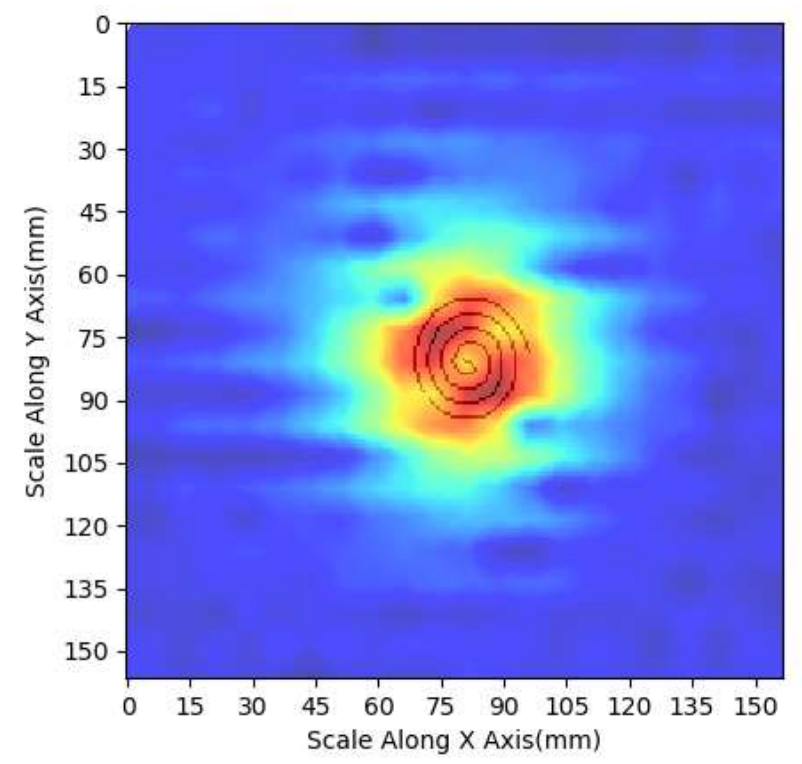

Fig. 5: Near field pattern in vertical plane with coil overlay.

The software also allows the interpolated measurements to be viewed as a full 3-D radiation pattern. To make the trends in the radiation pattern more perceivable, the 3-D matrix is thresholded such that voxels that fall below the programmed value are set to zero so that they become transparent when the image is rendered. When this feature is selected, the user can adjust a scrollbar that sets the threshold value, to adjust the size of the image. Additionally, this mode has the option of adding the segmented image of the CUT below the pattern. Some examples of the full 3-D radiation patterns with different thresholding values are shown in Fig. 6.

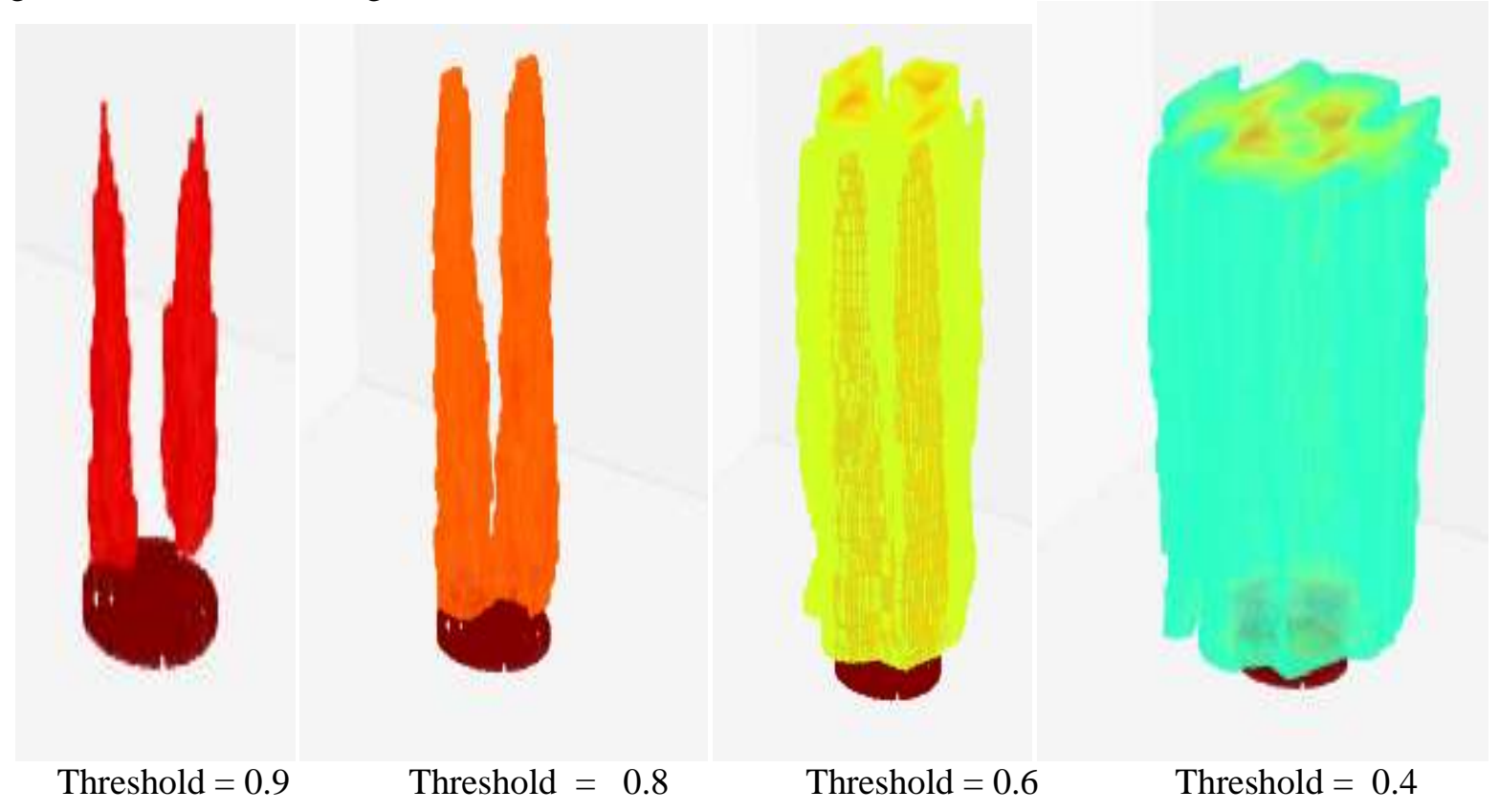

Fig. 6: 3-D radiation patterns with different threshold values.

As can be seen in the images present in Fig. 6, viewing the pattern at different thresholds provides a comprehensive view of the near field pattern. Furthermore, the resolution of these graphics can be increased to fit a more powerful computer than the one that this interface is intended for, which would create a smoother appearance to the 3-D patterns. 


\section{Conclusion}

The growing use of wireless power transfer in modern technology requires innovation of coil design and geometry. The interface presented in this paper provides improved graphics for comprehensive study on the effect that different coil

geometries have on the rendered near field pattern. The user interface and results presented can be used as a research tool for near field optimization. The multiple view perspectives allow a user to navigate the inherent complexity of the $\mathrm{H}$-fields and relate them to an overlaid outline of the CUT. Finally, the full 3-D plot provides the viewer with a visualization of an outward expansion of the radiation pattern by allowing configuration of the threshold voxel value.

\section{References}

[1] S. Li and C. C. Mi, "Wireless Power Transfer for Electric Vehicle Applications," IEEE Journal of Emerging and Selected Power Electronics, vol. 3, no. 1, pp. 4-17, March 2015.

[2] M. Feenaghty, "The Design of High-Quality Bifilar Archimdean Coil Geometries for Wireless Power Transfer Applications," M.S. Thesis, Dept. Elect. and Comp. Eng., State University of New York (SUNY) at New Paltz, New Paltz NY.

[3] M. Feenaghty and R. Dahle, "A compact and high-quality factor Archimedean coil geometry for wireless power transfer," 2016 IEEE Wireless Power Transfer Conference (WPTC), 2016.

[4] F. Las-Heras, M. R. Pino, S. Loredo, Y. Alvarez and T. K. Sarkar, "Evaluating Near-Field Radiation Patterns of Commercial Antennas," IEEE Transactions on Antennas and Propagation, vol. 54, no. 8, pp. 2198-2207, August 2006.

[5] M. Razmhosseini and R. G. Vaughan, "Aspects of Antenna Pattern Estimation from Planar Near-Fields," 2016 IEEE Canadian Conference on Electrical and Computer Engineering, 15-18 May 2016.

[6] C. Koenen, G. Hamberger, U. Siart and T. F. Eibert, "A Volumetric Near-Field Scanner for Millimeter-Wave Antenna Measurements," $201610^{\text {th }}$ European Conference on Antennas and Propagation, 10-15 April 2016.

[7] K. Sarabandi, J. Choi, A. Sabet and K. Sabet, "Pattern and Gain Characterization Using Nonintrusive Very-Near-Field Electro-Optical Measurements Over Arbitrary Closed Surfaces," IEEE Transactions on Antennas and Propagation, vol. 65, no. 2, pp. 489-497, February 2017.

[8] S. Muroga, K. Arai, S. Dhungana, R. Okuta, Y. Endo and M. Yamaguchi, "3-D Magnetic-Near-Field Scanner for IC Chip-Level Noise Coupling Measurements,” IEEE Transactions on Magnetics, vol. 49, no. 7, July 2013.

[9] MDL Techonologies. (2019, April 26). EMSCAN [Online].

Available: https://www.mdltechnologies.co.uk/manufacturers/emscan/

[10] R. Keys, "Cubic convolution interpolation for digital image processing." IEEE Transactions of Acoustics, Speech, and Signal Processing, vol. 29, pp. 1153 - 1160, December 1981.

[11] G. J. Grevera and J. K. Udupa, "Objective comparison of 3-D image interpolation methods," IEEE Transactions on Medical Imaging, vol. 17, pp. 642-652, August 1998.

[12] SciPy Developers. (2019, April 26). Scipy.org [Online]. Available: https://scipy.org/

[13] F. J. Blanco-Silva, Mastering SciPy. Birmingham UK: Packt Publishing Ltd., 2015, pp. 311-374. 